\title{
Modulated thermography of disbonds in the insulator of solid rocket motors
}

\author{
by Xingwang Guo*, Nannan Zhang*
}

* School of Mechanical Engineering and Automation, Beihang University, Beijing 100191, P. R. China, xingwangguo@buaa.edu.cn

\begin{abstract}
Solid rocket motors(SRMs) has been applied widely in missiles, space rockets and shuttles. However, because of some technology and environment reasons, disbonds or poor bonding usually appear in the insulator of solid rocket motors. It is necessary to find an effective nondestructive method to detect the disbonds. Modulated thermography(MT) has been used successfully for detecting defects in aerospace components due to its high efficiency and safe exploitation. In order to explore the optimal operation condition of applying MT to the inspection of SRM thermal insulators, the MT of disbonds in the insulator is studied through experimentation and 3D numerical simulation focusing on the influences of the modulation frequency, insulator thickness and disbond lateral size on defect signals. The results show that MT is an effective approach to detect the disbonds in the adhesion interfaces; the phase difference at the modulation frequency can be expressed as a quintic polynomial of the modulation frequency in the usual operation frequency range, and the best modulation frequency and detectable frequency band can be determined by the polynomial. When the insulator thickness increases, the best modulation frequency and the maximum phase difference decrease. When the disbond lateral size increases, the maximum phase difference increases, while the best modulation frequency remains unchanged. The results provide a guideline for the MT of disbonds in solid rocket motors.
\end{abstract}

Keywords: modulated thermography; solid rocket motor; insulator; disbonds

\section{Introduction}

A solid rocket motor(SRM) is mainly composed of a shell, insulator and propellant. The insulator is a polymer layer pasted on the inner wall of the shell to prevent it from being damaged by high-temperature and high-pressure gas. In addition, such insulator acts as a cushion for the stress transfer between the case and the propellant, and a seal for filament-wound composite cases, thus being an important part of SRMs. However, disbonds, or poor bonding in the adhesion interfaces, often appear during forming of the insulator because of technological reasons[1], thus possibly reducing the life-span of the SRM or leading to catastrophic failure of space vehicles. Thus, it's necessary to find an effective non-destructive testing (NDT) approach to detect the disbonds after insulator forming and before propellant filling. Ultrasonic inspection is usually used in the detection of interfacial debonding of SRMs even if it suffers from low productivity, contact operation and necessity for visual data interpretation. A severe drawback of the ultrasonic technique is that it cannot be applied to rocket motor cases made of filament-wound or braided composites, or covered with external thermal insulation, because these materials strongly scatter or attenuate ultrasonic waves. X-ray method is not applicable for kissing disbonds. Infrared thermography has been used successfully for detecting defects in aerospace components due to its high efficiency and safe exploitation[2-4]. According to the heat stimulation manner, there are different kinds of infrared thermographic approaches, including pulsed thermography(PT)[5] and modulated thermography(MT, also known as lock-in thermography)[6]. Some results of applying PT to the inspection of the thermal insulation of SRM cases had been reported elsewhere[7,8], and the result shows that it is feasible, but after all the limited energy of PT leads to its ineffectiveness when the insulator is thick. 


\subsection{1/qirt.2016.152}

MT has the advantage of low heating excitation and simple heating device. In order to explore the optimal operation condition of applying MT to the inspection of SRM thermal insulators, the MT of the disbonds in the insulator of SRMs is studied in this paper which focuses on the influences of the modulation frequency, insulator thickness and disbond lateral size on defect signals.

\section{Experiment}

\subsection{Sample and experimental setup}

Two identical samples were made to simulate insulator disbonds. The samples consist of an insulator made of nitrile-butadiene rubber (NBR), a substrate steel plate and an outside heat protection layer also made of NBR, as shown in Fig.1. Two groups of $0.08 \mathrm{~mm}$-thick polytetrafluoroethylene (PTFE) inserts with different shapes and sizes are embedded to imitate the disbonds. It is noteworthy that the inserts were moved in the direction away from the edge after they were placed, and in Fig.1, the dotted line represents the inserts position before being moved, the solid line stands for the position after being moved. The shifting of inserts produced two types of defects: a lack of adhesive and PTFE inserts.

An experiment with the sample was implemented in the infrared thermographic nondestructive testing laboratory of Beihang University. The experimental setup was composed by a computer, an IR camera, a control unit and a halogen lamp, as shown in Fig.2. The thermal resolution of the IR camera is $0.1 \mathrm{~K}$, and both the IR camera and the lamp are placed at the insulator side of the sample.

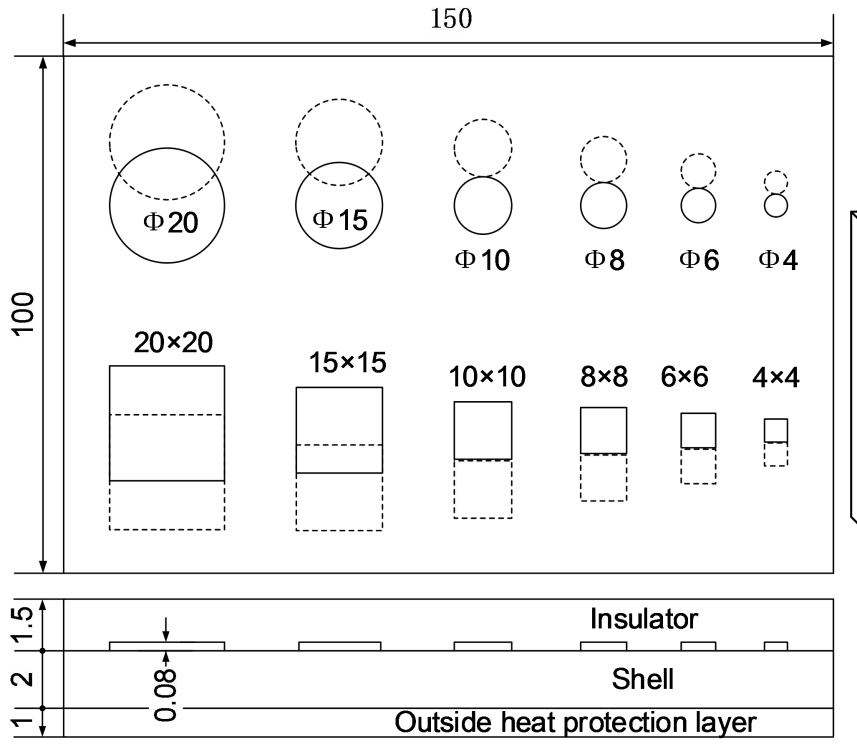

Fig. 1. Shape and size of the sample (all directions in $\mathrm{mm}$ )

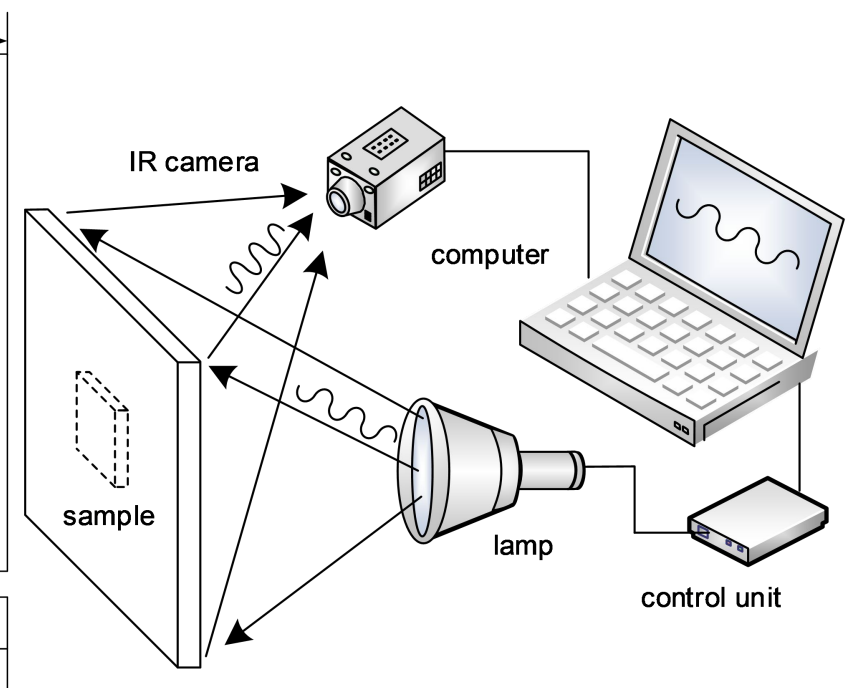

Fig. 2. Experimental setup

\subsection{Experimental analysis}

The sample was stimulated by the lamp with a sinusoidal modulated power. The modulation periods were set to $T=32 \mathrm{~s}, 48 \mathrm{~s}$ and $64 \mathrm{~s}$, and the corresponding sampling intervals were $2 \mathrm{~s}, 3 \mathrm{~s}$ and $4 \mathrm{~s}$, respectively. The thermal signals were recorded by the IR camera for several periods from the start of the fourth circle. A typical raw image taking at the time $t=122 \mathrm{~s}$ from the start of heating with $T=32 \mathrm{~s}$ is shown in Fig. $3 \mathrm{a}$, in which the artificial defects can be vaguely recognized. The cross and rectangle mark in Fig. $3 a$ stand for a defect area $(3 \times 3$ pixels $)$ and non-defect area $(5 \times 5$ pixels $)$ of interest respectively, and the corresponding temperature evolution are shown in Fig.3b. The phase difference and phase contrast spectrum produced by the Fast Fourier Transform (FFT) of the signals in Fig.3b are shown in Fig.3c, d, which display that 


\subsection{1/qirt.2016.152}

both the phase difference and phase contrast reach its maximum value(absolute value) at the modulation frequency $\left(f_{\mathrm{M}}=1 / T=0.0313 \mathrm{~Hz}\right)$, so the modulation frequency is a key parameter in MT method.

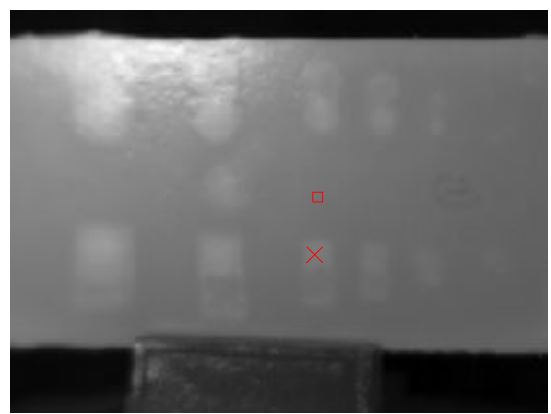

(a)

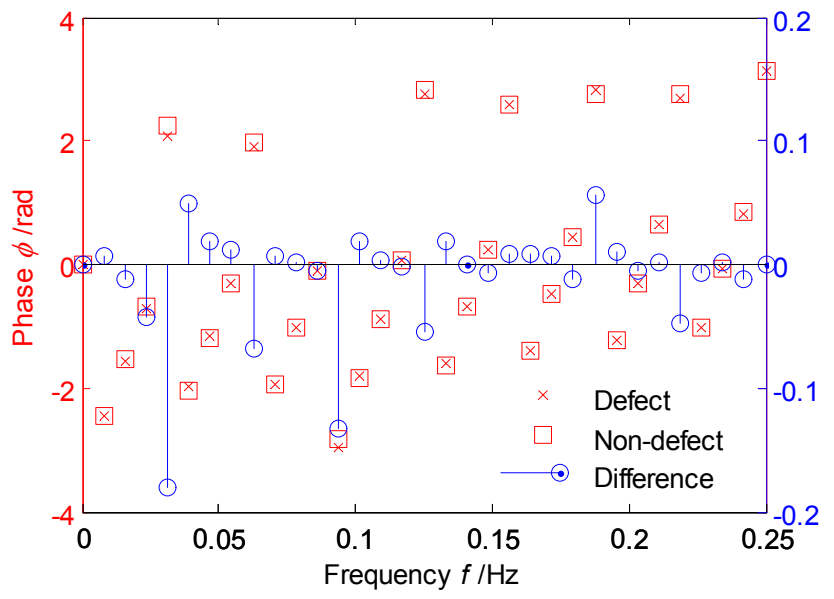

(c)

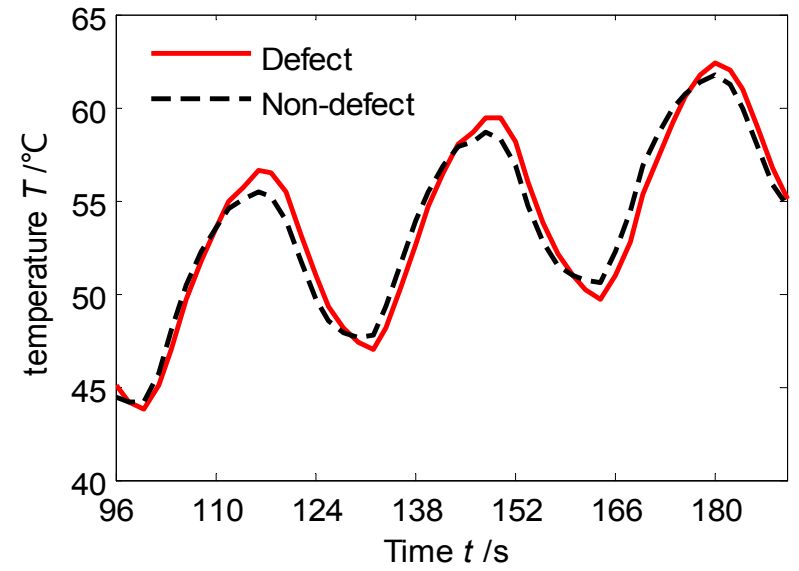

(b)

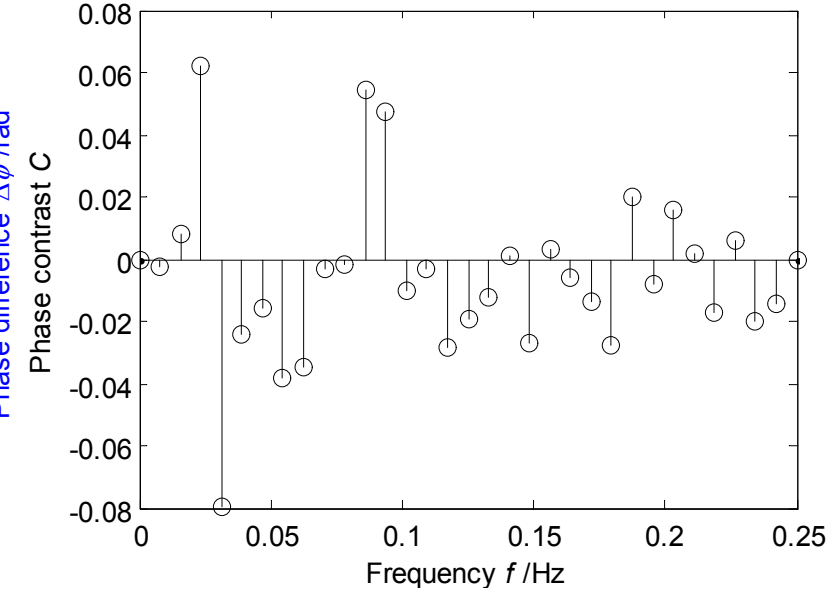

(d)

Fig.3. Data analysis: a-raw image with marks of defect and non-defect areas; b-temperature vs. time; c-phase and phase difference spectrum; $d$-phase contrast spectrum

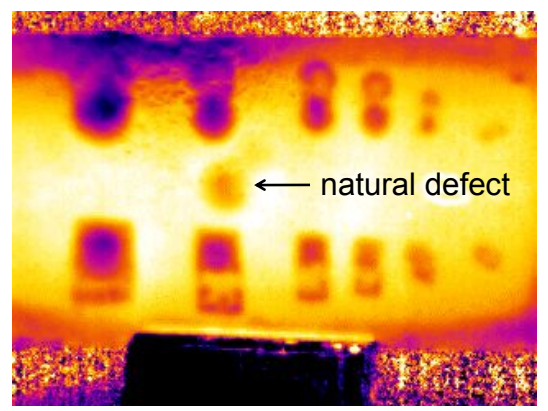

(a)

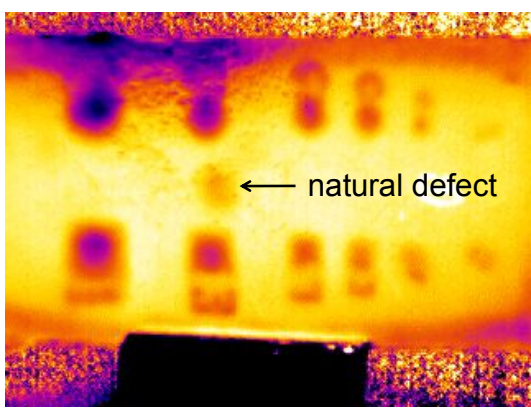

(b)

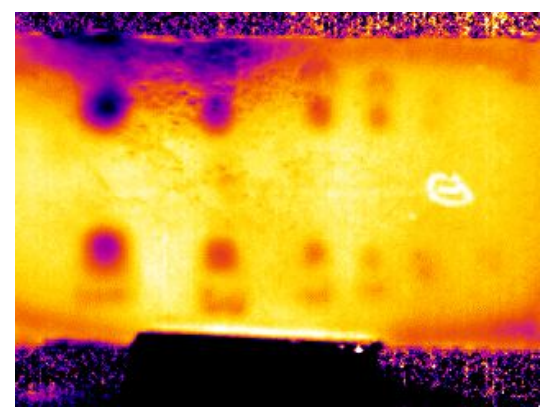

(c)

Fig.4. Phase images at different modulation frequencies: $a-f_{\mathrm{M}}=0.0313 \mathrm{~Hz}, T=32 \mathrm{~s} ; b-f_{\mathrm{M}}=0.0208 \mathrm{~Hz}, T=48 \mathrm{~s}$;

$$
c-f_{\mathrm{M}}=0.0156 \mathrm{~Hz}, T=64 \mathrm{~s}
$$




\subsection{1/qirt.2016.152}

The phase images at the modulation frequencies for $T=32 \mathrm{~s}, 48 \mathrm{~s}$ and $64 \mathrm{~s}$ are shown in Fig.4, which were obtained by using the FFT of the thermal image sequences. The defects become clearer in phase images(Fig.4a) than in raw image(Fig.3a). Among these three phase images in Fig.4, the defects in Fig.4a is the clearest, so the corresponding modulation frequency $0.0313 \mathrm{~Hz}$ is more close to the best modulation frequency. A natural defect can also be seen in Fig.4a, b.

\section{Numerical simulation}

There are many physical parameters that influence the disbond detectability. The influences of the modulation frequency, disbond lateral size and insulator thickness on the defect signals are basic guidelines of optimizing the test conditions. To explore the test regularities experimentally, a lot of reference samples are needed. In order to avoid manufacturing too many samples and conducting too many experiments, 3D numerical simulation method was adopted[8,9].

\subsection{Simulation model}

To speed up the calculation, each defect was modelled separately. Two models were used to describe the heat conduction of the local area of a square disbond defect, as shown in Fig.5, where only a quarter of the area was selected due to the symmetrical nature of the structure, $L$ denotes the disbond size, point $D$ and $N$ denote the center of the defect and non-defect areas on the upper surface, respectively. A $0.05 \mathrm{~mm}$-thick air gap is used to instead the $0.08 \mathrm{~mm}$-thick PTFE insert, because the insert leads to a kind of hybrid defect due to the presence of air gaps between the insert and the host material, and the hybrid defect can be thermally detected in the same way as a pure air gap, which was confirmed elsewhere $[8,10]$. The only difference of the two models is whether or not there is a bulge on the upper surface. In model $\mathrm{A}$ (Fig.5a), the upper surface of the defect area is slightly bulged and the thickness of the bulge is the same as that of the air gap. To simplify the model, the bulge is ignored in model B (Fig.5b). The thermal properties of related materials is listed in table 1.

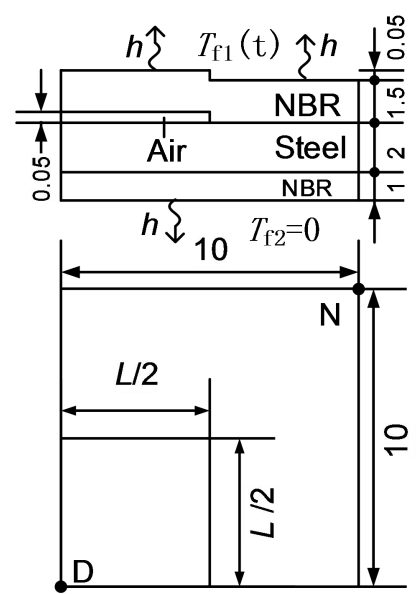

(a) Model A

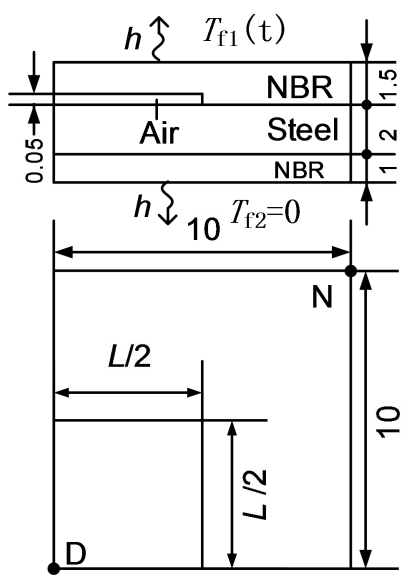

(b) Model B

Fig.5. Simulation models (all dimensions in $\mathrm{mm}$ ) 
Table 1. Thermal properties of the materials

\begin{tabular}{cccc}
\hline Material & Density $\rho / \mathrm{Kg} \mathrm{m}^{-3}$ & Specific heat $c / \mathrm{J} \mathrm{Kg}^{-1} \mathrm{~K}^{-1}$ & Thermal conductivity $\mathrm{k} / \mathrm{W} \mathrm{K} \mathrm{K}^{-1} \mathrm{~m}^{-1}$ \\
\hline Insulator(NBR) & 1070 & 1694 & 0.448 \\
Steel & 7900 & 440 & 46 \\
Air & 1.2 & 700 & 0.02 \\
PTFE & 2140 & 1050 & 0.24 \\
\hline
\end{tabular}

The numerical simulation was performed using the software Ansys 15.0, which runs a finite element analysis scheme. The two models were evaluated under the same conditions: the environment temperature of the front(upper) surface is changed as Eq. (1), while the environment temperature of the rear(lower) surface keeps at zero.

$$
T_{\mathrm{f} 1}(t)=\frac{q_{0}}{2}\left(1-\cos \left(2 \pi f_{\mathrm{M}} t\right)\right)
$$

where $q_{0}$ is the peak value of the thermal wave, $f_{\mathrm{M}}$ is the modulation frequency. The heat exchange coefficient $h$ on both the front and rear surface is $30 \mathrm{Wm}^{-1} \mathrm{~K}^{-1}$ (this value is intentionally enlarged so that the thermal response of the model can reach its quasi-steady period early); the element type used is Solid 70, and the simulation step is set to $0.5 \mathrm{~s}$. The phase information at the modulation frequency for model $A$ and $B$ are compared in table 2 , which shows that the relative difference of the phase difference and phase contrast of the two models are small and within $3 \%$. The reason is that the bulge is very thin. If the bulge is thicker, then the difference will be larger. For the samples discussed here, both of the two models are adopted. Model B is used in the following analysis.

Table 2. Phase information at the modulation frequency for the two models and their comparison

\begin{tabular}{lcccc}
\hline & Model A & Model B & Absolute difference & $\begin{array}{c}\text { Relative difference } \\
\text { (Value of model A as reference) }\end{array}$ \\
\hline Phase difference $\Delta \Phi / \mathrm{rad}$ & -0.2053 & -0.2006 & 0.0047 & $2.29 \%$ \\
Phase contrast $C$ & -0.0747 & -0.0730 & 0.0017 & $2.28 \%$ \\
\hline
\end{tabular}

\subsection{Influences of modulation frequency on defect signals}

The phase difference and phase contrast between the defect and non-defect areas at the modulation frequency are the most significant defect signals. The phase difference and phase contrast at different modulation frequencies $(f=0.0125-0.1 \mathrm{~Hz})$ obtained by simulation and experiment for the defect with size $L=10 \mathrm{~mm}$ are shown in Fig.6. The distribution of the phase difference and the phase contrast in the modulation frequency domain are called the phase difference spectrum and the phase contrast spectrum respectively in this paper, and the two spectrums are also called defect signal spectrums generally.

Both of the absolute values of the simulation defect signals increase first and then decrease with the modulation frequency increasing, and take on a ' $V$ ' shape, so a best modulation frequency exists, at which the defect signals can reach their maximum values. In order to get a better detectability, the modulation frequency should be as close as possible to the best modulation frequency in practice. 
Fig.6 also shows the experimental defect signals at the modulation frequency $f_{\mathrm{M}}=0.0313 \mathrm{~Hz}, 0.0208 \mathrm{~Hz}$ and $0.0156 \mathrm{~Hz}$, and the difference between the simulation and experimentation results is small.

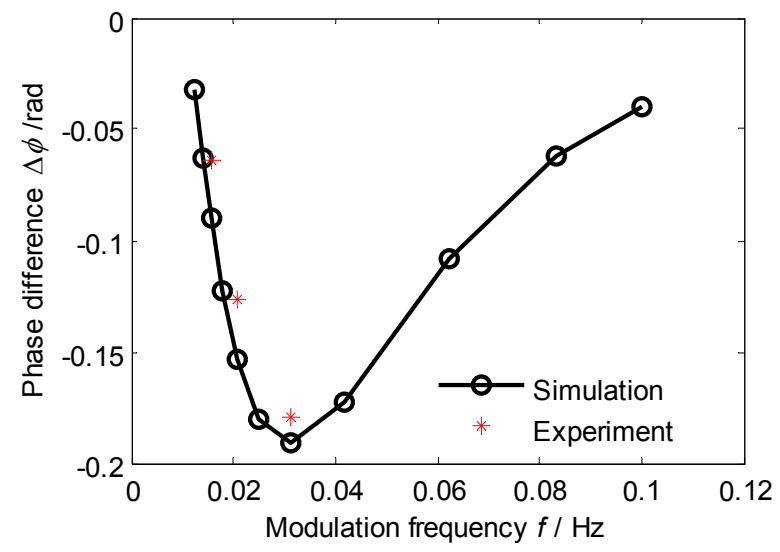

(a)

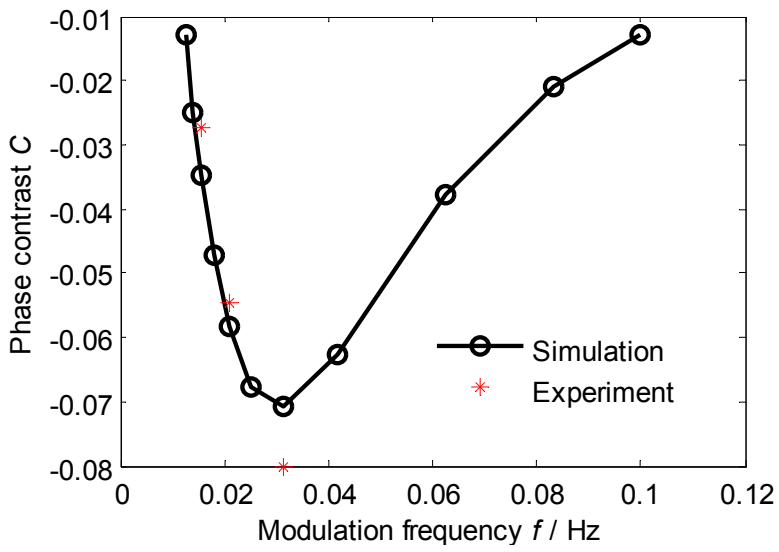

(b)

Fig.6. Influences of modulation frequency on defect signals: a-phase difference vs. modulation frequency; b-phase contrast vs. modulation frequency

\subsection{Influences of insulator thickness on defect signals}

The insulator thickness is different in different SRMs or at different places of one SRM. Therefore, it is necessary to research the influences of insulator thickness on the defect signal spectrums. The simulation results with the insulator thickness of $1.5 \mathrm{~mm}, 2 \mathrm{~mm}$ and $2.5 \mathrm{~mm}$ are shown in Fig.7, which depicts that the thicker the insulator is, the smaller the maximum phase difference and the maximum phase contrast are, and the lower the best modulation frequency is.

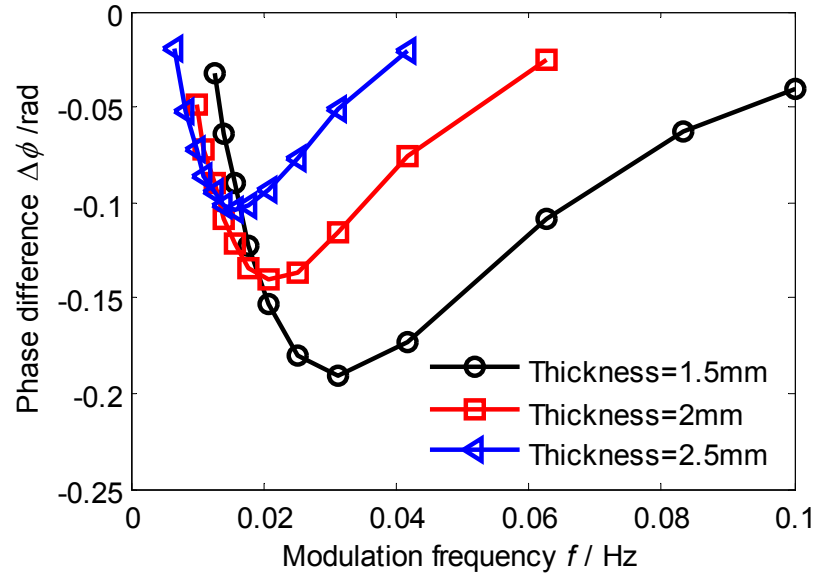

(a)

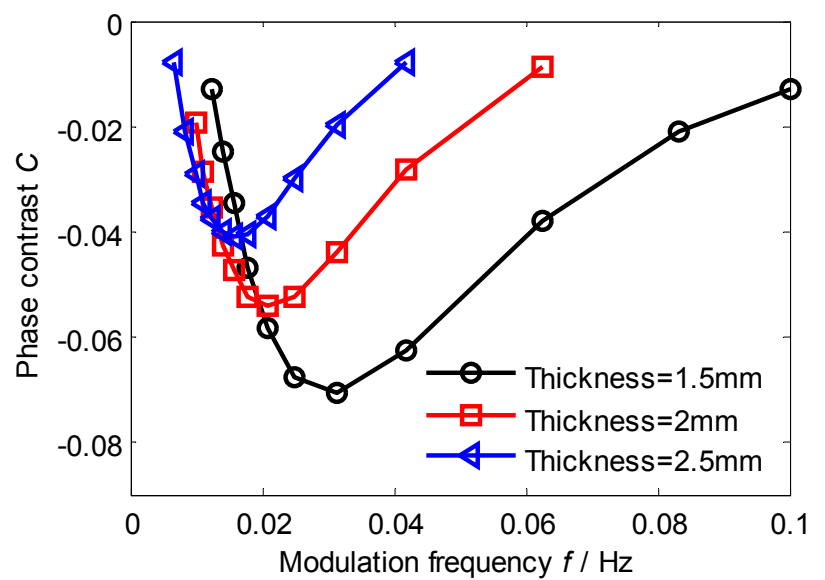

(b)

Fig.7. Influences of insulator thickness on defect signal spectrums: a-phase difference spectrum; $b$-phase contrast spectrum

The regression equations between the phase difference and the modulation frequency when the insulator thickness $(H)$ is $1.5 \mathrm{~mm}, 2 \mathrm{~mm}$ and $2.5 \mathrm{~mm}$ are Eqs.(2), (3) and (4), respectively, which were generated by fitting the simulation data as shown in Fig.8 (with the same data in Fig.7a). The phase difference corresponding to any modulation frequency can be achieved easily with these equations, the best modulation frequency $f_{\mathrm{m}}$ and the corresponding 
maximum phase difference $\Delta \phi_{\mathrm{m}}$ are listed in table 3.

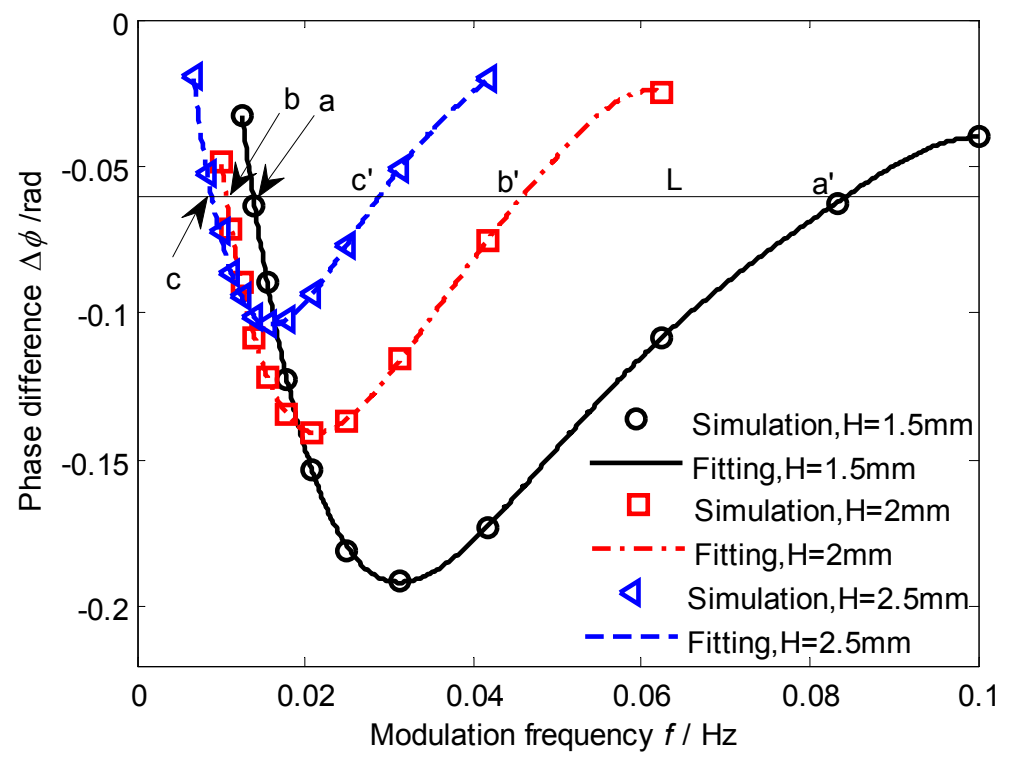

Fig.8. Fitting curves of the phase difference data for different insulator thicknesses

$\Delta \phi_{1.5}=-6.149 \times 10^{5} f^{5}+2.129 \times 10^{5} f^{4}-2.889 \times 10^{4} f^{3}+1898 f^{2}-57.07 f+0.4351, \quad(0.0125 \leq f \leq 0.1)$

$\Delta \phi_{2}=-4.202 \times 10^{6} f^{5}+9.030 \times 10^{5} f^{4}-7.790 \times 10^{4} f^{3}+3343 f^{2}-67.05 f+0.3563,(0.01 \leq f \leq 0.0625)$

$\Delta \phi_{2.5}=-6.135 \times 10^{6} f^{5}+1.267 \times 10^{6} f^{4}-9.906 \times 10^{4} f^{3}+3645 f^{2}-59.34 f+0.2415,(0.0067 \leq f \leq 0.0417)$

Table 3. The best modulation frequency and maximum phase difference gotten from the fitting polynomials

Insulator thickness $H(\mathrm{~mm}) \quad$ The best modulation frequency $f_{\mathrm{m}} \quad(\mathrm{Hz}) \quad$ The maximum phase difference $\Delta \phi_{\mathrm{m}} \quad(\mathrm{rad})$

\begin{tabular}{ccc}
\hline 1.5 & 0.0313 & -0.1917 \\
2 & 0.0214 & -0.1407 \\
2.5 & 0.0160 & -0.1039 \\
\hline
\end{tabular}

Let us define a suitable phase threshold for defect recognition based on the experimental phase images in Fig. 4. Choose a 30×30-pixel non-defect area in the phase images, the phase standard deviation $\sigma$ of the chosen area at the modulation frequency can be obtained, which is $0.0061,0.0030$ and $0.0042 \mathrm{rad}$, corresponding to the case of $T=32 \mathrm{~s}, 48 \mathrm{~s}$ and $64 \mathrm{~s}$ respectively. The biggest one is $\sigma_{\mathrm{m}}=0.0061 \mathrm{rad}$. A threshold of $P_{\mathrm{th}}=10 \sigma_{\mathrm{m}}=0.06$ rad are adopted considering the uneven heating and other noises. When the absolute phase difference is larger than the threshold, the defect can be recognized reliably on the phase image.

In Fig.8, set a threshold line $\mathrm{L}$ as $\Delta \phi=-P_{\text {th }}$, then the line intersects the fitting curves at the point pairs [a, a'], [b, b'], [c, c'] respectively. The frequency range between [a, a'], [b, b'] or [c, c'] is defined as the detectable frequency band, 
and only the modulation frequency is within it, can the defect be detected reliably. The range and width of the detectable frequency bands are shown in table 4, which shows that the detectable frequency band for a thick insulator covers a small range, in other words, it is more difficult to select an effective modulation frequency in actual operation when the insulator is thicker.

Table 4. Range and width of the detectable frequency band

\begin{tabular}{ccc}
\hline Insulator thickness $H(\mathrm{~mm})$ & Range of detectable frequency band $(\mathrm{Hz})$ & Width of detectable frequency band $(\mathrm{Hz})$ \\
\hline 1.5 & $0.0138-0.0845$ & 0.0707 \\
2 & $0.0106-0.0459$ & 0.0353 \\
2.5 & $0.0089-0.0289$ & 0.0200 \\
\hline
\end{tabular}

\subsection{Influences of disbond lateral size on defect signals}

The simulation defect signal spectrums with the disbond lateral size $L=6 \mathrm{~mm}, 8 \mathrm{~mm}$ and $10 \mathrm{~mm}$ are shown in Fig.9, which explains that the best modulation frequency of different disbond lateral size is very close, but the larger the disbond is, the bigger the maximum phase difference and the maximum phase contrast are. In other words, disbonds with different lateral sizes will get the best detection effect at the same modulation frequency, but the larger one will be clearer in phase images. The simulation results explain the fact that all the defects shown in Fig.4a are clearer than those shown in Fig. $4 \mathrm{~b}, \mathrm{c}$, and the largest disbond $(L=20 \mathrm{~mm}$ and $\Phi=20 \mathrm{~mm})$ is the clearest.

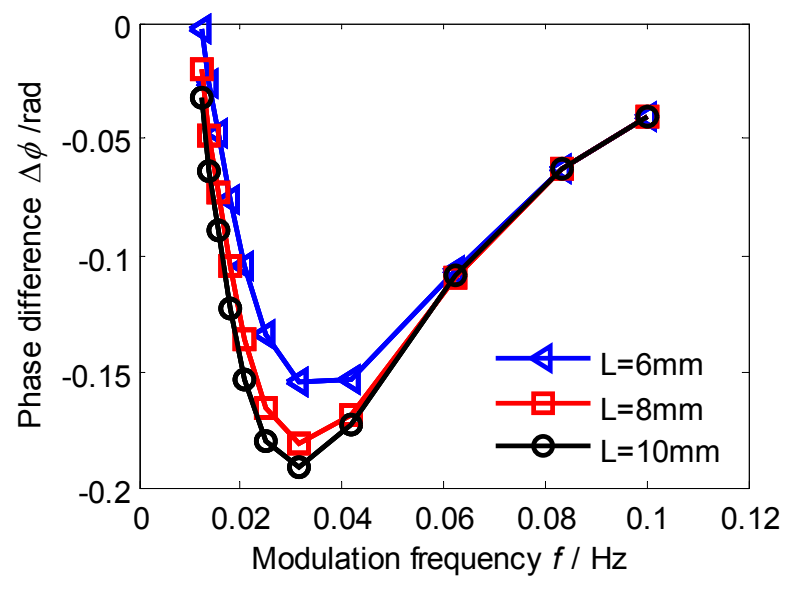

(a)

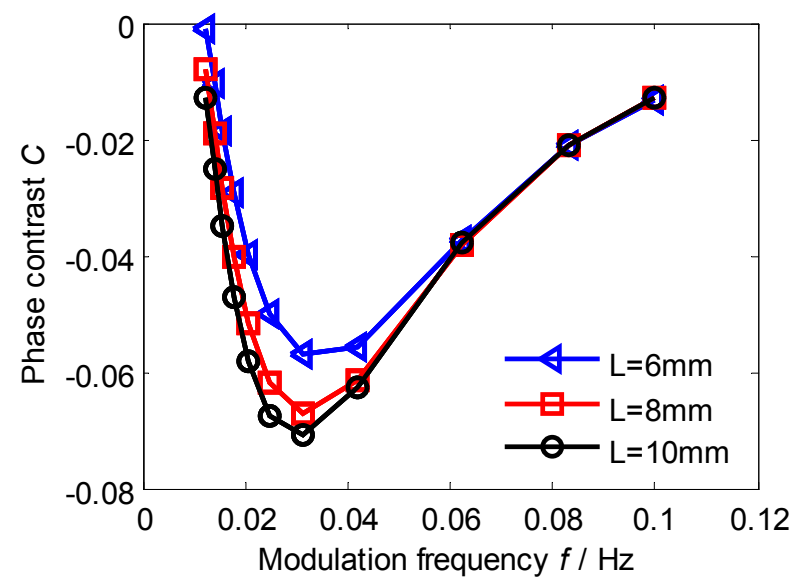

(b)

Fig.9. Influences of disbond lateral size on defect signals: a-phase difference spectrum; b-phase contrast spectrum

The regression equations between the phase difference and modulation frequency when the disbond lateral size is $6 \mathrm{~mm}, 8 \mathrm{~mm}$ and $10 \mathrm{~mm}$ are Eqs.(5), (6) and (7), respectively, which were generated by fitting the simulation data as shown in Fig.10 (with the same data in Fig.9a). The best modulation frequency $f_{\mathrm{m}}$ and the corresponding maximum phase difference $\Delta \phi_{\mathrm{m}}$ are listed in table 5. In Fig.10, the threshold line $\mathrm{L}\left(\Delta \phi=-P_{\mathrm{th}}\right)$ which intersects the fitting curves at the point pairs $\left[A, A^{\prime}\right],\left[B, B^{\prime}\right],\left[C, C^{\prime}\right]$ are also depicted. The corresponding range and width of the detectable frequency bands are listed in table 6 , which shows that the disbond lateral size has little impact on the range and width of the detectable frequency band. 


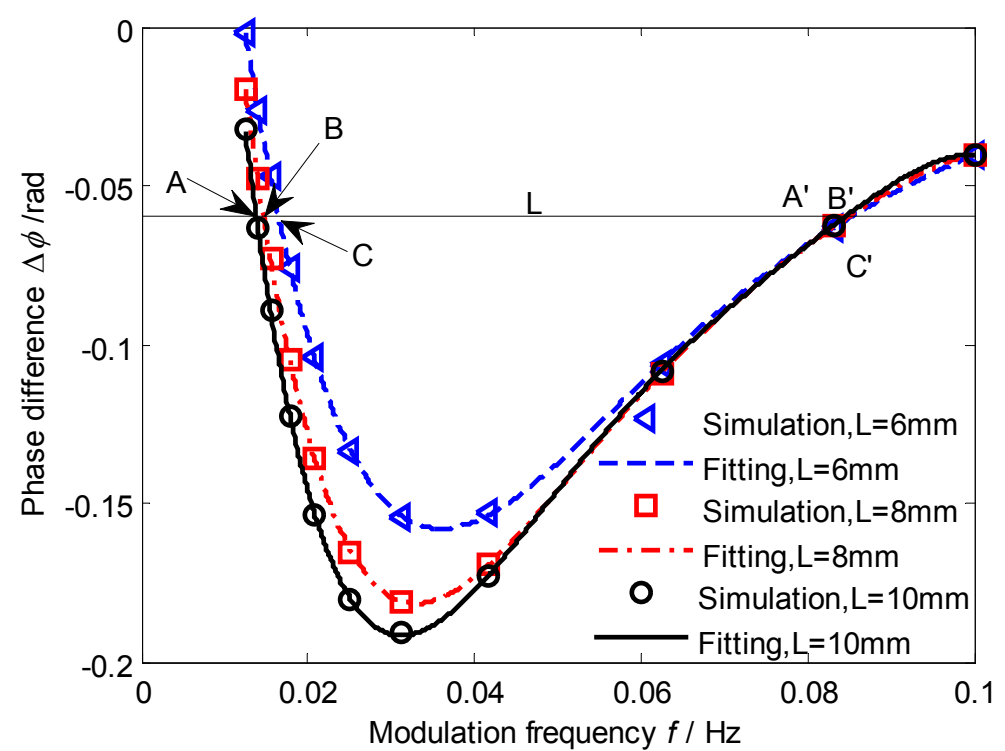

Fig.10. Fitting curves of the phase difference data for different disbond lateral sizes

$$
\begin{aligned}
& \Delta \phi_{6}=-2.006 \times 10^{5} f^{5}+8.265 \times 10^{4} f^{4}-1.343 \times 10^{4} f^{3}+1058 f^{2}-37.67 f+0.3279, \quad(0.0125 \leq f \leq 0.1) \\
& \Delta \phi_{8}=-4.219 \times 10^{5} f^{5}+1.540 \times 10^{4} f^{4}-2.219 \times 10^{4} f^{3}+1555 f^{2}-49.77 f+0.3992, \quad(0.0125 \leq f \leq 0.1) \\
& \Delta \phi_{10}=-6.149 \times 10^{5} f^{5}+2.129 \times 10^{5} f^{4}-2.889 \times 10^{4} f^{3}+1898 f^{2}-57.07 f+0.4351, \quad(0.0125 \leq f \leq 0.1)
\end{aligned}
$$

Table 5. The best frequency and maximum phase difference gotten from the fitting polynomials

Defect lateral size $L(\mathrm{~mm}) \quad$ The best modulation frequency $f_{m}(\mathrm{~Hz}) \quad$ The maximum phase difference $\Delta \phi_{m}(\mathrm{rad})$

\begin{tabular}{ccc}
\hline 6 & 0.0361 & -0.1580 \\
8 & 0.0331 & -0.1816 \\
10 & 0.0313 & -0.1917 \\
\hline
\end{tabular}

Table 6. The scope and width of threshold band

\begin{tabular}{ccc}
\hline Defect lateral size $L(\mathrm{~mm})$ & Range of detectable frequency band $(\mathrm{Hz})$ & Width of detectable frequency band $(\mathrm{Hz})$ \\
\hline 6 & $0.0165-0.0852$ & 0.0687 \\
8 & $0.0147-0.0849$ & 0.0702 \\
10 & $0.0138-0.0845$ & 0.0707 \\
\hline
\end{tabular}

\section{Conclusions}

(1) Both the experimental and simulation results prove that MT is an effective approach to detect the disbonds between the insulator and shell of solid rocket motors.

(2) When the insulator thickness increases, the best modulation frequency decreases, the maximum phase 


\subsection{1/qirt.2016.152}

difference becomes small and the detectable frequency band becomes narrow, so a disbond under a thicker insulator is more difficult to detect.

(3) The best modulation frequency depends on the thickness of the insulator, and is independent of the lateral size of the disbond; the maximum phase difference increases with the disbond lateral size, so a larger defect is easier to detect.

(4) The phase difference at the modulation frequency can be expressed as a quintic polynomial of the modulation frequency in the usual operation frequency range, and the best modulation frequency and detectable frequency band can be determined by the polynomial.

These results provide a guideline for the MT of disbonds in the insulator of solid rocket motors.

\section{Acknowledgment}

This work is supported by NSFC under project: 61571028, U1433122.

\section{REFERENCES}

[1] Wang W, Zhang F. Analysis and preventative of debonds between case and insulation in solid rocket motors. Journal of Propulsion Technology, 1996, 17(1): 89-91. (in Chinese)

[2] Vavilov V P, Burleigh D D. Review of pulsed thermal NDT: Physical principles, theory and data processing. NDT\&E International, 2015, 73: 28-52.

[3] Ibarra-Castanedo C, Piau J-M, Guilbert S, Avdelidis N, Genest M, Bendada A, Maldague X. Comparative study of active thermography techniques for the nondestructive evaluation of honeycomb structures. Research in Nondestructive Evaluation, 2009, 20: 1-31.

[4] Meola C, Carlomagno G M, Squillace A, Vitiello A. Non-destructive evaluation of aerospace materials with lock-in thermography. Engineering Failure Analysis, 2006, 13: 380-388.

[5] Maldague X, Marinetti S. Pulse Phase Infrared Thermography. Journal of Applied Physics, 1996,79(5):2694-2698.

[6] Busse G, Wu D, Karpen W. Thermal wave imaging with phase sensitive modulated thermography, J. Appl. Phys. 1992 71 (8): 3962-3965

[7] Guo X, Vavilov V. Pulsed thermographic evaluation of disbonds in the insulation of solid rocket motors made of elastomers. Polymer Testing, 2015, 45: 31-40.

[8] Jiang S, Guo X, Shen J, Zhang C. Infrared thermal wave NDT on the disbonds of the heat insulated layer in solid propellant rocket motors. Laser and Infrared, 2005, 35(8):584-586. (in Chinese)

[9] Guo X, Qie F, Simulating thermal NDT of bonded structures by FEM. In: Vavilov V P, Burleigh D D. Proc. SPIE, Thermosense XXX, Bellingham, WA: SPIE, 2008, 6939: 69391H.

[10] Vavilov V P, Burleigh D D, Klimov A G. Advanced modeling of thermal NDT problems: from buried landmines to defects in composites. In: Maldague X P, Rozlosnik A E. Proc. SPIE, Thermosense XXIV, Bellingham, WA: SPIE, 2002, 4710: 507-521. 